\title{
Sistema de Suporte à Decisão baseado em Lógica Fuzzy para Outorga de Recursos Hídricos Superficiais
}

\author{
Carlos Ueslei R. de Oliveira ${ }^{1}$, Peter Zeilhofer ${ }^{1}$ \\ ${ }^{1}$ Programa de Pós-Graduação em Física Ambiental- Universidade Federal do Mato \\ Grosso (UFMT)
}

Av. Fernando Corrêa da Costa, 2367 - 78060-900 - Cuiabá - MT - Brasil

carlos@ic.ufmt.br, zeilhoferpeter@gmail.com

\begin{abstract}
The growing demand for water resources has forced managers to create control mechanisms to ensure the sustainability of water sources and manage conflicts among users. In this paper we present the development of a Decision Support System based on fuzzy logic and AHP to evaluate the effects of multiple uses in watersheds on water availability considering quantitative and qualitative characteristics of water. It also allows the generation of scenarios to solve possible conflicts, distributing the burden for maintenance of the hydrological system between users designed and already installed in a basin. Design and operation of the prototype and its outputs are illustrated.

Resumo. A crescente demanda por recursos hídricos tem obrigado os gestores a criarem mecanismos de controle para garantir a sustentabilidade dos mananciais e gerir conflitos entre usuários. Neste trabalho está exposto o desenvolvimento de um Sistema de Suporte à Decisão baseado em lógica fuzzy e AHP para avaliar os efeitos dos usos múltiplos em bacias hidrográficas sobre a disponibilidade hídrica considerando características quantitativas $e$ qualitativas da água. Possibilita também a geração de cenários para solução de possíveis conflitos, distribuindo o ônus para manutenção do sistema hidrológico entre usuários projetados e já instalados em uma bacia. Concepção e funcionamento do protótipo e suas saídas são ilustrados.
\end{abstract}

\section{Introdução}

A água é um elemento indispensável para a manutenção da vida e, apesar da sua abundância sobre a superfícies do planeta, somente uma pequena fração de $0,007 \%$ da água potável se encontra em rios e lagos de onde é retirada a maior parte para usos consuntivos [USGR 2016].

A partir de 1980 a captação mundial de água doce tem aumentado cerca de $1 \%$ ao ano, principalmente devido à crescente demanda dos países em desenvolvimento. [UNESCO 2016]. O aumento da retirada da água de rios e lagos para usos consuntivos e o aumento do lançamento de efluentes não devidamente tratados têm pressionado a 
sustentabilidade desses corpos d'água prejudicando não só o meio ambiente como as comunidades humanas localizadas à sua jusante [Oliveira, 2009].

O Brasil, apesar de ter em geral alta disponibilidade de água doce, da expressiva heterogeneidade hidroclimática, da distribuição demográfica e do desenvolvimento agrícola e industrial, passa por problemas regionais de escassez de disponibilidade em termos quantitativos e/ou qualitativos, podendo gerar conflitos entre usuários. Isso fez com que fosse instalada em 1997 a Política Nacional de Recursos Hídricos (Lei $\mathrm{n}^{\circ}$ 9.433), seguida pelas respectivas legislações estaduais, responsável por legislar a gestão da água.

Uma das ferramentas utilizadas para o controle do uso é chamada "Outorga de Recursos Hídricos", um contrato firmado entre uma unidade concedente (Órgão Gestor) e um consumidor. No caso da Outorga de águas superficiais é estipulada uma vazão máxima a ser retirada em um determinado trecho de um rio durante um determinado tempo, o chamado uso direto. Caso um consumidor pretenda lançar efluentes é também estipulada uma vazão necessária para depurar esse efluente, vazão essa chamada de uso indireto. A soma dos usos diretos e indiretos de um determinado trecho junto com a soma de todos os usos à montante não podem afetar a vazão ecológica, uma vazão mínima previamente estipulada pelo gestor e necessária para que o rio se mantenha sustentável.

A utilização desses contratos junto com a devida fiscalização ajuda ao Órgão Gestor a ter um maior controle sobre os mananciais, evitando assim possíveis conflitos pelo uso de seus recursos hídricos, mas como gerenciar conflitos quando a vazão disponível para Outorga já está quase que totalmente concedida e o Órgão Gestor recebe novos pedidos de concessão que comprometeriam a vazão ecológica? A simples negação da concessão fará com que o Órgão Gestor tenha problemas de natureza política devido aos impactos socioeconômicos que essa negação trará para as populações afetadas por essa decisão.

Uma forma de resolver esse problema seria diminuir as vazões concedidas em contratos já assinados no mesmo trecho ou em trechos a montante de onde essa entidade deseja se instalar. Essa solução é possível devido ao fato de que o Órgão Gestor tender a ser menos rigoroso nos primeiros contratos de Outorga e também pelo fato de as entidades solicitantes, precavendo-se frente a possíveis aumentos de demanda de vazão no futuro, solicitem uma vazão relativamente maior que a necessária atualmente. $\mathrm{O}$ Órgão Gestor poderia dessa forma diminuir sensivelmente a quantidade de vazão contratada de um grupo de usuários, sem prejudicar as suas atividades, de forma a sobrar uma vazão resultante em quantidade suficiente para ser usado por um novo usuário sem prejudicar a sustentabilidade do rio.

A quantidade de alternativas válidas para solucionar esse problema torna complicada a tarefa do Órgão Gestor em tomar a melhor decisão que satisfaça tanto a sustentabilidade do rio quanto ao conjunto de usuários afetados. Um Sistema de Suporte à Decisão (SSD) pode ser usado para analisar todas as alternativas existentes segundo regras estabelecidas pelo Órgão Gestor e apresentar uma lista com as melhores 
alternativas existentes, auxiliando assim o Órgão Gestor a tomar a melhor decisão possível.

Nesse contexto, o presente trabalho propõe o desenvolvimento de um SSD que visa disponibilizar uma ferramenta para analisar a disponibilidade hídrica em mananciais superficiais, avaliar a contribuição dos usuários no comprometimento dos recursos hídricos ao longo da bacia e propor, a partir de critérios múltiplos hierarquizados, soluções para compartilhar a disponibilidade hídrica entre usuários concorrentes.

O sistema proposto utiliza a lógica fuzzy como técnica para avaliar se as alternativas avaliadas cumprem as regras definidas pelo Órgão Gestor e o método AHP (Analytic Hierarchy Process) para categorizar qual regra é mais importante em relação as demais.

\section{O Sistema desenvolvido}

O sistema Web aqui demonstrado foi desenvolvido usando a ferramenta case Genexus na sua versão 9, gerando o código em Java e acessando uma base de dados PostGreSql 8 .

O sistema foi dividido em dois componentes (Figura 1). O primeiro é o módulo da outorga, no qual é possível analisar cada trecho de um determinado rio para identificar quais vazões já foram concedidas e quanto ainda existe de vazão disponível a ser distribuída para novos empreendimentos.

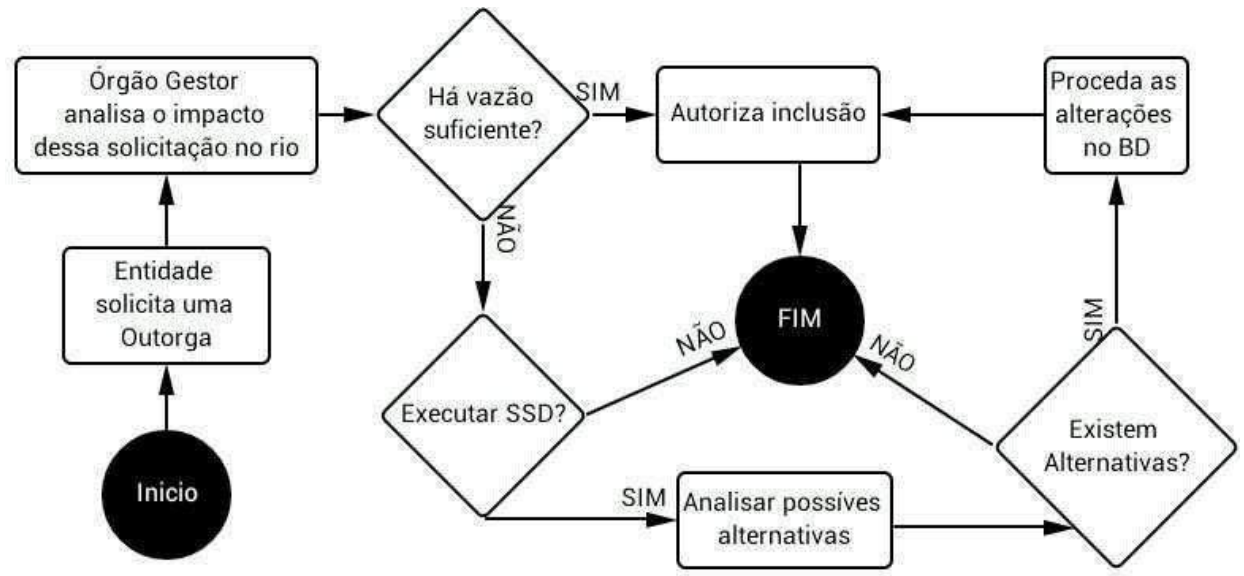

Figura 1 - Fluxograma do funcionamento do Sistema de Outorga proposto

A outra parte é o módulo do SSD que somente é acionado quando a simulação de inserção de um novo empreendimento apresentar um quadro de déficit de vazão em algum dos trechos do rio.

\subsection{O Módulo de Outorga}

Este módulo foi desenvolvido baseado na metodologia apresentada por Da Hora (2001) que calcula a vazão $\mathrm{v}$ ainda disponível para outorga em um determinado trecho através da Equação 1. 


$$
v_{d}(i, t)=v^{+}(i, t)-\sum_{j=\epsilon_{M}}\left(u(j) \cdot v(j, t)+v_{q}(j, t)\right)+\sum_{j=\epsilon_{M}}(1-u(j)) \cdot v(j, t) \quad \text { [Equação 1] }
$$

Ou seja, a vazão disponível em um determinado trecho i de um rio em um determinado tempo t, representada por $v_{d}(i, t)$, será a diferença entre a vazão máxima disponível para outorga nesse trecho/tempo, representada por $v^{+}(i, t)$, e a soma de todos os usos diretos (captação) e indiretos (lançamento de efluentes) da água dos empreendimentos existentes desde a montante $M$ do rio até esse trecho. A soma dos usos diretos e indiretos em nenhum momento poderá ser maior que a vazão disponível para todo trecho i e em todo tempo t.

Uma primeira validação do sistema foi realizada a partir de um conjunto de dados referentes a bacia do Rio Coxipó, afluente da margem esquerda do rio Cuiabá (código ANA 6610000), que drena partes do perímetro urbano da cidade de Cuiabá, capital de Mato Grosso e que possui usuários múltiplos com captações e lançamentos de efluentes de diversas origens (indústria, abastecimento público etc.). Figura 2 mostra a situação de alguns trechos do rio Coxipó com a sua vazão média mínima (Vazão em $\mathrm{m}^{3} / \mathrm{s}$ ) - no caso a denominada $\mathrm{Q}_{95}$, que representa a vazão que está presente no rio durante, pelo menos, $95 \%$ do tempo. Outros campos representam as concentrações da Demanda Biológica de Oxigênio (DBO), a vazão máxima disponível a ser outorgada, o número de empreendimentos e as captações e lançamentos acumulados nos respectivos trechos.

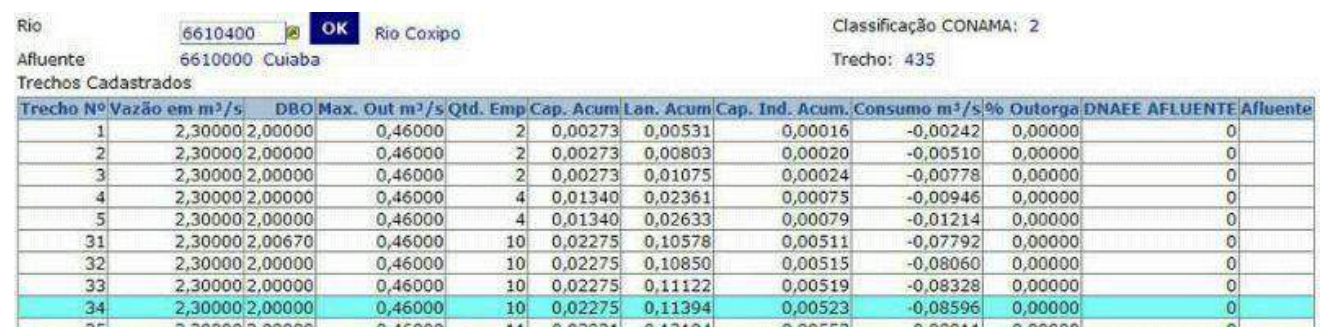

Figura 2 - Tela do Sistema mostrando a vazão estimada em alguns trechos do rio Coxipó

Quando um novo empreendimento desejar se instalar em um determinado trecho ele deverá informar ao Órgão Gestor qual será a vazão de retirada necessária e, se houver lançamento de efluentes, a vazão de lançamento do efluente com a concentração de DBO para cálculo da vazão de uso indireto desse empreendimento. De posse desses valores o sistema simula o impacto desse novo empreendimento em um determinado trecho do rio. Se em nenhum trecho, desde o trecho em que esse empreendimento deseja instalar-se até a jusante do rio, houver uma vazão requerida maior que a vazão disponível será permitida a sua inclusão. Uma vez incluído no sistema, os seus dados serão levados em consideração para cálculos posteriores.

Caso o sistema encontre um ou mais trechos entre o trecho desejado e a jusante com vazão requerida maior que a vazão disponível, o sistema mostra os trechos com problemas destacando o trecho com maior déficit de vazão (Figura 3). 


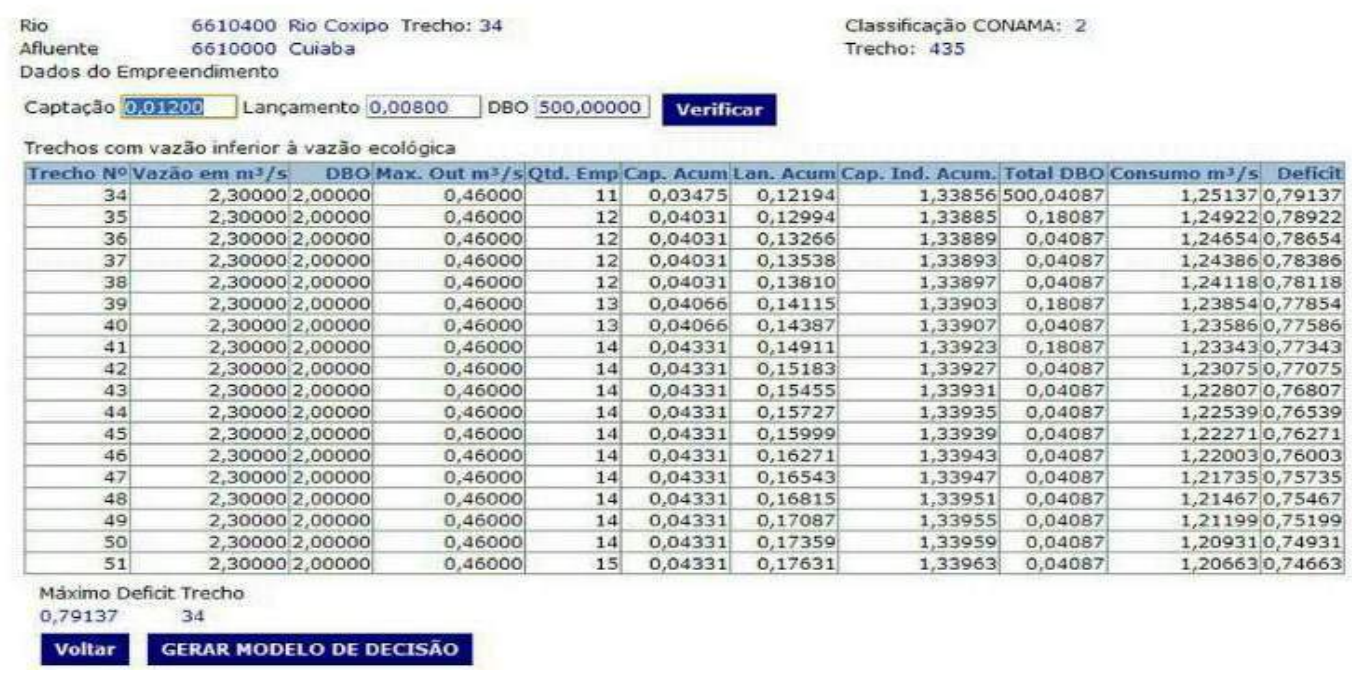

Figura 3 - Tela do sistema mostrando características de alguns trechos do rio Coxipó e identificação do trecho com o maior déficit de vazão.

Nesse exemplo, o trecho com maior déficit de vazão é justamente o trecho no qual o empreendimento deseja se instalar, porém, pode acontecer de o trecho de maior déficit se encontra a jusante deste trecho.

O sistema dessa forma permite ao Órgão Gestor decidir sobre a Outorga solicitada. A Outorga pode ser negada para esse empreendimento sob a justificativa que isso afetaria a sustentabilidade do rio a partir daquele trecho, ou o modelo de SSD pode ser inicializado que mostrará outras alternativas que permitam a inclusão desse empreendimento mantendo a sustentabilidade do rio.

\subsection{O Módulo de Sistema de Suporte à Decisão}

O módulo de Suporte a Decisão tem como objetivo apresentar ao Órgão Gestor uma lista com no máximo 10 agrupamentos de entidades, com no máximo 5 membros cada situados a montante do trecho com maior déficit e que poderiam ter suas vazões sensivelmente diminuída. Esses agrupamentos são então ranqueados de acordo com parâmetros definidos anteriormente pelo Órgão Gestor. Com essa lista de agrupamentos o Órgão Gestor pode decidir qual agrupamento terá suas vazões diminuídas e em qual quantidade para cada membro, liberando assim vazão suficiente para que possa incluir a entidade requisitante da Outorga.

O módulo de suporte a decisão foi implementado utilizando modelo de decisão fuzzy proposto por O'Hagan (1993) para inter-relacionar objetivos e critérios do problema na busca pela sua melhor solução. O’Hagan (1993) demonstrou que combinando as operações de união, intersecção e exponenciação é possível construir mecanismos de suporte a tomada de decisão que permitam analisar cada uma das alternativas apresentadas frente aos critérios objetivos e as restrições para a solução do problema proposto.

Para Bellman e Zadeh (1970), uma boa alternativa deve satisfazer tanto os 
objetivos quanto às restrições de certo problema. Já uma alternativa ótima será aquela que melhor satisfaça todos os critérios no sentido comum. Eles sugeriram uma regra de conjunção implícita com $m$ objetivos e $\mathrm{n}$ restrições de um problema que avalia uma determinada alternativa através de uma intersecção pela fórmula demonstrada na Equação 2.

$$
\mu_{G 1}(x) \cap \mu_{G 2}(x) \cap \ldots \mu_{G i}(x) \cap \mu_{C 1}(x) \cap \mu_{C 2}(x) \cap \ldots \mu_{C j}(x)
$$

[Equação 2]

Onde $\mu_{\mathrm{Gi}}(\mathrm{x})$ é o valor difuso para o i-ésimo objetivo avaliado para a alternativa $\mathrm{x}$ e $\mu_{\mathrm{Cj}}(\mathrm{x})$ é o valor difuso para a j-ésima restrição para a alternativa x. Para resolver essa conjunção é preciso utilizar o operador MIN sobre todos os valores difusos da alternativa $x$ resultando em um novo conjunto difuso $\mathrm{D}(\mathrm{x})$ para a decisão que satisfaça tanto os objetivos quanto às restrições do problema, de acordo com Equação 3.

$$
D(x)=\operatorname{Min}\left(\mu_{G 1}(x), \ldots, \mu_{G i}(x), \mu_{C 1}(x), \ldots, \mu_{C j}(x)\right)
$$

[Equação 3]

$\mathrm{O}$ conjunto difuso $\mathrm{D}(\mathrm{x})$ será definido sobre todas as alternativas $\mathrm{x} \in \mathrm{X}$. Para uma ótima tomada de decisão é preciso selecionar a alternativa $\mathrm{x}^{*}$ que terá o mais alto valor de pertinência no conjunto de decisões $\mathrm{D}(\mathrm{x})$. A alternativa $\mathrm{x}^{*}$ será aquela que melhor irá satisfazer o conjunto de objetivos e restrições para o problema proposto. Essa alternativa pode ser definida pela Equação 4.

$$
x^{*}=\operatorname{Max}[D(x)] x \in X
$$

Uma limitação da metodologia proposta por Bellman e Zadeh (1970) é que ela pressupõe que todos os objetivos e restrições do problema possuem o mesmo grau de importância, o que é algo difícil de ser verificado no mundo real. Para resolver essa limitação Yager (1977) sugeriu elevar os valores de pertinência dos conjuntos de objetivos e restrições por um peso que representasse a importância de um determinado objetivo ou restrição frente aos demais para a solução do problema. O peso ajudaria a definir processos de decisão de conjuntos difusos mais representativos para as necessidades do mundo real. Dessa forma a função de decisão é definida como demonstrado pela Equação 5.

$$
D(x)=\operatorname{Min}\left(\mu_{G 1}^{\alpha 1}(x), \ldots, \mu_{G i}^{\alpha m}(x), \mu_{C 1}^{\alpha m+1}(x), \ldots, \mu_{C j}^{\alpha m+n}(x)\right)
$$

[Equação 5]

Sendo $\alpha$ o peso que avalia o grau de importância do objetivo m ou da restrição n.

Para mensurar a relativa importância entre os objetivos ou restrições em problemas complexos e assim obter os seus respectivos pesos, Saat (1977) propôs um método de análise multicritério do tipo interativo chamado Analytic Hierarchy Process (AHP), modelo que permite estruturar hierarquicamente qualquer problema com múltiplos critérios, múltiplas decisões e múltiplos períodos. É um processo flexível, que apela para a lógica e ao mesmo tempo, utiliza a intuição. [Orofino, 1996] 
Uma estruturação hierárquica demanda primeiramente a definição dos critérios que serão utilizados para a solução do problema. Posteriormente é necessário fazer a comparação de cada par de critérios gerados na etapa anterior, obtendo dessa forma o grau de importância de um critério em relação ao outro para a análise de solução do problema. Ou seja, dado um critério e duas alternativas A e B, qual alternativa que mais satisfaz, e quanto mais em relação ao critério considerado? Para tal julgamento, é utilizada uma escala de referência de 1 a 9, tal como mostrado na Tabela 1.

\section{Tabela 1 - Grau de importância entre um determinado objetivo/restrição e os} outros.

\begin{tabular}{ll}
\hline Julgamento & Valores \\
\hline X é igualmente preferivel a Y & 1 \\
X é igulmente a moderadamente preferivel sobre Y & 2 \\
X é moderadamente preferivel sobre Y & 3 \\
X é moderadamente a fortemente preferivel sobre Y & 4 \\
X é fortemente preferivel sobre Y & 5 \\
X é fortemente a muito fortemente preferivel sobre Y & 6 \\
X é muito fortemente preferivel sobre Y & 7 \\
X é muito fortemente a extremamente preferivel sobre Y & 8 \\
X é extremamente preferivel sobre Y & 9
\end{tabular}

Os resultados obtidos com os julgamentos, através da comparação paritária são colocados numa matriz A quadrada $\mathrm{n} \times \mathrm{n}$. Este procedimento se repete para todos os elementos do nível, com respeito a todos os elementos de um nível acima.

A matriz de comparação A para comparar n elementos (a) é demonstrada na Equação 6.

$A=\left\lfloor a_{i j}\right\rfloor\left(\right.$ onde $\left.a_{j i}=\frac{1}{a_{i j}}, a_{i i}=1,1 \leq i \leq n, e 1 \leq j \leq n\right)$

[Equação 6]

A diagonal principal é sempre 1. Nota-se a reciprocidade através da diagonal, ou seja, se o elemento $\mathrm{a}_{12}=3$, então $\mathrm{a}_{21}=1 / 3$.

De acordo com Betencourt (2000), o próximo passo é calcular o peso relativo das alternativas com respeito aos critérios. Os pesos relativos são obtidos através da aplicação de um processo de duas etapas. Primeiro, soma-se cada coluna e, então, se divide cada coluna entrada pela soma respectiva de cada coluna. A matriz que resulta do processo é chamada de matriz normalizada. (Equação 7)

$$
A^{\prime}=\left\lfloor a_{i j}^{\prime}\right\rfloor \text { onde } a_{i j}^{\prime}=\frac{a_{i j}}{\sum_{k=1}^{n} a_{k j}} \text { para } 1 \leq i \leq n \text {, e } 1 \leq k \leq n
$$

Depois, calcula-se o valor médio de cada linha da matriz normalizada para obter o peso relativo ou eigenvector, o qual é determinado pela Equação 8.

$$
W=\left[w_{k}\right] \text { onde } w_{k}=\frac{\sum_{j=1}^{n} a_{i j}^{\prime}}{n} \text { para } 1 \leq j \leq n \text {, e } 1 \leq k \leq n
$$

O eigenvector é o vetor de pesos a ser utilizado para acrescentar o grau de importância de cada objetivo ou restrição frente ao seu conjunto.

No SSD desenvolvido foram criados os seguintes parâmetros a serem usados 
como pesos na função de decisão:

- $\quad$ Percentual da captação em relação a Vazão máxima disponível para Outorga Esse parâmetro visa diferenciar aquelas entidades que retiram uma grande quantidade de água do rio daquelas que tiram pouco.

- $\quad$ Concentração de DBO - Parâmetro usado para beneficiar aquelas entidades que lançam efluentes já devidamente tratados evitando assim o uso da vazão de depuração utilizada pelo rio.

- $\quad$ Tempo médio de contrato - Esse parâmetro tende a diferenciar as entidades que recém adquiriram os contratos de Outorga daquelas que já estão com seus contratos perto de expirarem.

Esses parâmetros estão disponíveis ao Órgão Gestor em uma tela de configuração como a apresentada na Figura 4. Nessa, o gestor deve selecionar qual a relativa importância de cada um dos critérios na decisão da concessão de Outorga por múltiplos objetivos/restrições.

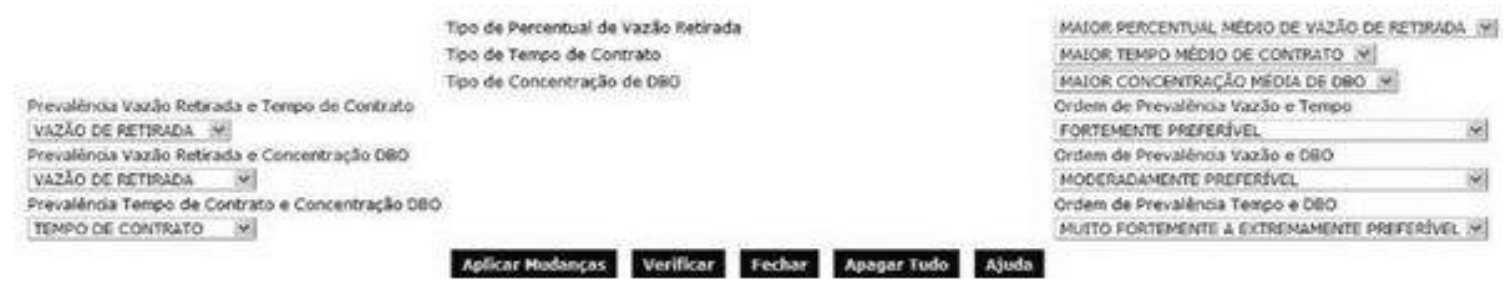

Figura 4 - Tela de configuração de preferência de objetivos/restrições para a geração do vetor de pesos

Pela prevalência relativa, os três pares de parâmetros são comparados. A vazão de retirada pode ter por exemplo uma importância relativa maior do que a concentração de DBO e uma menor do que o Tempo de contrato. A configuração desejada vai depender das características existentes na região da bacia. Como exemplo um Órgão Gestor de uma Bacia Hidrográfica pode querer incluir na análise preferencialmente aqueles usuários com maior tempo médio de contrato, mais próximos de uma eventual renovação e não incluir preferencialmente aqueles que recém efetivaram os seus contratos de outorga. Já em outra bacia o Órgão Gestor pode querer incluir os usuários com menos tempo de contrato de outorga, pois os mesmos podem ainda não estar utilizando o total de vazão retirada concedida. Por último, pode ser configurada a relação entre os três pares de parâmetros a partir de nove graus de importância (Tabela 1). O conjunto dessas configurações orienta o módulo de SSD na análise das alternativas geradas e classificá-las, permitindo ao gestor tomar a melhor decisão para o problema apresentado.

Assim que o módulo SSD é acionado, o sistema irá buscar uma lista com todos os usuários de outorga cadastrados a montante do trecho com problema e criará até 2000 grupos com até 5 usuários cada onde a soma da diminuição da vazão de cada um deles seja suficiente para manter a vazão ecológica do rio. Esses grupos depois serão analisados e categorizados seguindo as configurações pré-definidas (Figura 4) e as 10 
melhores alternativas são apresentadas ao gestor para que ele possa decidir qual grupo será escolhido para possibilitar a inclusão do novo usuário.

A Figura 5 mostra o resultado de uma simulação realizada pelo sistema, mostrando os grupos formados que poderiam ter suas vazões diminuídas para a inclusão de uma nova entidade.

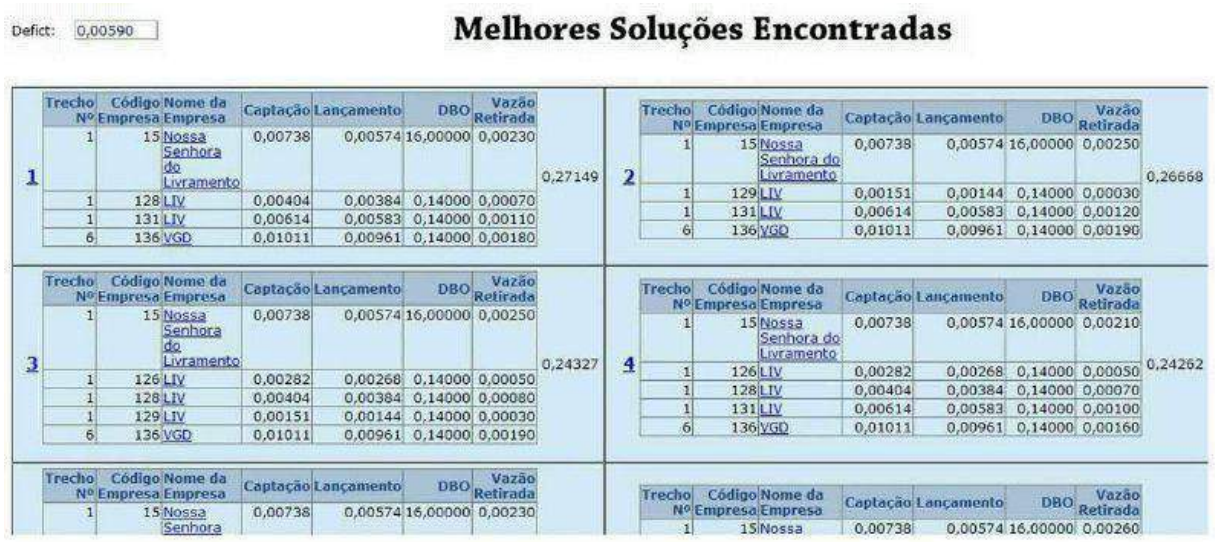

Figura 5 - Tela com o resultado de uma simulação mostrando as melhores alternativas de diminuição da vazão outorgada, possibilitando a inclusão de um novo usuário.

Nessa tela são expostas para cada grupo de usuários as seguintes informações:

- Índice com a posição desse grupo em relação aos demais;

- Trecho do rio na qual se encontra cada um dos usuários do grupo;

- Código do usuário no sistema;

- Nome do usuário;

- Vazão de captação (uso direto) de cada usuário, acordada no contrato de outorga;

- Vazão de lançamento de efluentes (uso indireto) de cada usuário, acordada no contrato de outorga;

- Quantidade de DBO lançado por cada usuário no grupo via efluentes

- Vazão sugerida de retirada para cada usuário desse grupo para contribuir para a solução do problema;

- Soma das vazões sugeridas de retirada de cada grupo.

Com essas informações o gestor pode escolher qualquer um dos grupos apresentados ao escolher o link situado no número referente ao grupo desejado. Efetuada a escolha, o sistema processa as alterações diminuindo as vazões de retirada em cada um dos usuários do grupo escolhido nos valores de retirada sugeridos pelo sistema e finaliza incluindo o novo usuário no sistema.

\section{Conclusão}

O uso do Sistema desenvolvido fornece aos gestores de recursos hídricos novas possibilidades na tomada de decisão na concessão de Outorga, problema que pode se 
tornar altamente complexo em bacias com diversos usos concorrentes, impossibilitando uma análise objetivo da situação por técnicos nos órgãos gestores. Sendo baseado em modelos matemáticos e dados reais, decisões dos gestores na concessão se tornam reproduzíveis, aspecto relevante pelo fato das legislações nacionais e estaduais não fixar detalhadamente os critérios da Outorga e por poder produzir consequências legais na (não-)concessão dos direitos de uso. A implementação proposta possibilitará, por sua estrutura modular, futuramente adaptar o processo decisório para prioridades distintas de conservação em função das características das bacias em questão (urbano-industrial, rural etc.) e seus problemas específicos como p.ex. alta produção de sedimentos, baixa qualidade de água, excesso de captação para irrigação etc.

\section{Referências}

Da Hora, A. F. (2001) "Metodologia para a outorga do uso de recursos hídricos com ênfase no caso de aproveitamento hídrico", Tese de Doutorado. Coordenação dos Programas de Pós-Graduação de Engenharia da Universidade Federal do Rio de Janeiro - COPPE/UFRJ. Rio de Janeiro.

O’Hagan, Michael (1993) “A fuzzy decision maker”, Harcourt Brance \& Cia.

Bellman,R.E. and Zadeh, L.A. .(1970). "Decision Making in a Fuzzy Environment", Management Science, 17,141-164.

Yager, R.R. (1977)."Multiple Objective Decision-Making Using Fuzzy Sets," Intl. J.Man-Machine Studies, 9, 375-382.

Saaty, T.L. (1977). "A Scaling Method for Priorities in Hierarchical Structures," J.Math. Psychology, 15,234-281

Orofino, F. V. G. (1997) “Aplicação de um Sistema de Suporte Multicritério Saat For Windows - Na Gestão dos Resíduos Sólidos de Serviços de Saúde - Caso do Hospital Celso Ramos". Dissertação de Mestrado. Universidade Federal de Santa Catarina.

Betencourt, PAULO R. B. (2000) "Desenvolvimento de um modelo de análise multicriterial para justificativa de investimentos em tecnologia da informação". Dissertação de Mestrado em Administração na UFRGS.

USGR. (2016) “The World's Water”. http://water.usgs.gov/edu/earthwherewater.html, February

UNESCO (2016) "Relatório Mundial das Nações Unidas sobre Desenvolvimento dos Recursos Hídricos 2016 - $\quad$ Relatório Executivo". http://unesdoc.unesco.org/images/0024/002440/244040por.pdf, February

Oliveira, C. U. R. "Sistema de Suporte à Decisão Baseado em Lógica fuzzy para Outorga de Recursos Hídricos". Cuiabá, 2009. 72p. Dissertação (Mestrado) Programa de Pós-Graduação em Física Ambiental, Universidade Federal de Mato Grosso. 